\title{
Innate gene signature distinguishes humoral versus cytotoxic responses to influenza vaccination
}

\author{
Eléna Gonçalves, ${ }^{1}$ Olivia Bonduelle, ${ }^{1}$ Angèle Soria, ${ }^{1,2}$ Pierre Loulergue, ${ }^{3}$ Alexandra Rousseau, ${ }^{4}$ Marine Cachanado,${ }^{4}$ \\ Henri Bonnabau, ${ }^{5}$ Rodolphe Thiebaut, ${ }^{5}$ Nicolas Tchitchek, ${ }^{6}$ Sylvie Behillil, ${ }^{7,8}$ Sylvie van der Werf, ${ }^{7,8}$ Annika Vogt, ${ }^{1,9}$ \\ Tabassome Simon, ${ }^{4}$ Odile Launay, ${ }^{3}$ and Behazine Combadière ${ }^{1}$
}

'Sorbonne Université, Centre d'Immunologie et des Maladies Infectieuses - Paris (Cimi-Paris), INSERM U1135, Paris, France. Service de Dermatologie et Allergologie, Hôpital Tenon, Assistance Publique Hôpitaux de Paris (AP-HP), Paris, France. JUniversité Paris Descartes, Sorbonne Paris Cité, Centre d'Investigation Clinique Cochin Pasteur, INSERM CIC 1417, French Clinical Research Infrastructure Network, Innovative Clinical Research Network in Vaccinology, AP-HP, Hôpital Cochin, Paris, France. Department of Clinical Pharmacology and Clinical Research Platform of East of Paris, Assistance Publique-Hôpitaux de Paris, Paris, France. Sorbonne Université, Paris, France. ${ }^{5}$ INSERM U1219, INRIA SISTM, Université de Bordeaux, Bordeaux France. ${ }^{6}$ CEA - Université Paris Sud 11 - INSERM U1184, Immunology of Viral Infections and Autoimmune Diseases, Institut de Biologie François Jacob, 92265 Fontenay-aux-Roses, France. IInstitut Pasteur, CNR des Virus des Infections Respiratoires, Département de Virologie and Centre National de Recherche Scientifique UMR CNRS 3569, Paris, France. ${ }^{8}$ Université Paris Diderot, Sorbonne Paris Cité, Unité de Cénétique Moléculaire des Virus à ARN, Paris, France. ${ }^{9}$ Clinical Research Center for Hair and Skin Science, Department of Dermatology and Allergy, Charité-Universitätsmedizin Berlin, Berlin, Germany.

BACKGROUND. Systems vaccinology allows cutting-edge analysis of innate biomarkers of vaccine efficacy. We explored a strategy to shape the adaptive immune response by targeting innate immune cells through novel immunization routes.

METHODS. This randomized phase I/II clinical study ( $n=60$ healthy subjects aged $18-45$ years old) used transcriptomic analysis to discover early biomarkers of immune response quality after transcutaneous (t.c.), intradermal (i.d.), and intramuscular (i.m.) administration of a trivalent influenza vaccine (TIV, season 2012-2013, 1:1:1 ratio). Safety and immunogenicity (hemagglutinin inhibition [HI], microneutralization [MN] antibodies, and CD4+ and CD8+ effector T cells) were measured at baseline day $\mathrm{O}(\mathrm{dO})$ and at d21. Blood transcriptome was analyzed at d0 and d1.

RESULTS. TIV-specific CD8+ granzyme B+ (GRZ) T cells appeared in more individuals immunized by the t.c. and i.d. routes, whereas immunization by the i.d. and i.m. routes prompted high levels of $\mathrm{HI}$ antibody titers and MN against A/H1N1 and A/H3N2 influenza viral strains. The early innate gene signature anticipated immunological outcome by discriminating 2 clusters of individuals with either distinct humoral or CD8 cytotoxic responses. Several pathways explained this dichotomy and confirmed that 9 genes and the serum level of CXCL10 were correlated with either TIV-specific cytotoxic CD8+CRZ+ T cell or antibody responses. A logistic regression analysis demonstrated that these 9 genes and the serum levels of CXCL10 at d1/d0 best predicted TIV-specific CD8+GRZ+ T cell and antibody responses at $\mathrm{d} 21$.

CONCLUSION. This study provides new insight into the impact of immunization routes and innate signature in the quality of adaptive immune responses.

TRIAL REGISTRATION. This study has been registered at ClinicalTrials.gov (NCT01707602).

FUNDING. This work was supported by grants from the French Ministry of Health PHRCN 2012 - RCT 12061, INSERM-DCOS, the Fondation pour la Recherche Médicale, and the Société Française de Dermatologie (to AS). These funding sources had no direct role in any aspect of the research or article.

\section{Introduction}

Because innate immunity plays an important role in adaptive immune response to vaccination, researchers have been explor-

Authorship note: EG, OB, and AS contributed equally to this work. Conflict of interest: BC has belonged to advisory boards of Sanofi Pasteur MSD. SVW has received support from GlaxoSmithKline for meeting attendance and attended sponsor-funded meetings. PL and OL have participated as investigators in vaccine studies sponsored by Sanofi Pasteur MSD, Janssen, and GlaxoSmithKline, and have attended sponsor-funded meetings.

Copyright: (c) 2019, American Society for Clinical Investigation.

Submitted: October 10, 2018; Accepted: March 1, 2019; Published: April 8, 2019.

Reference information: J Clin Invest. 2019;129(5):1960-1971.

https://doi.org/10.1172/JCl125372. ing novel strategies to shape the adaptive immune response by targeting innate immune cells through the use of adjuvants, viral vectors, or novel immunization routes $(1,2)$. Specifically, adjuvant formulation and alternative routes of immunization have sought to increase the immunogenicity of influenza vaccination and improve immune protection, or even induce cellular immunity in immunocompromised individuals (3-5). Besides the standard intramuscular (i.m.) vaccination route, which provides moderate and variable protection against influenza mainly through humoral response, intradermal (i.d.) and transcutaneous (t.c.) routes have been proposed to induce cellular immunity $(1,6,7)$. Clinical trials assessing t.c. methods, such as that developed by our group targeting vaccine at hair follicles, have revealed that they induce a dif- 
CONSORT 2010 flow diagram

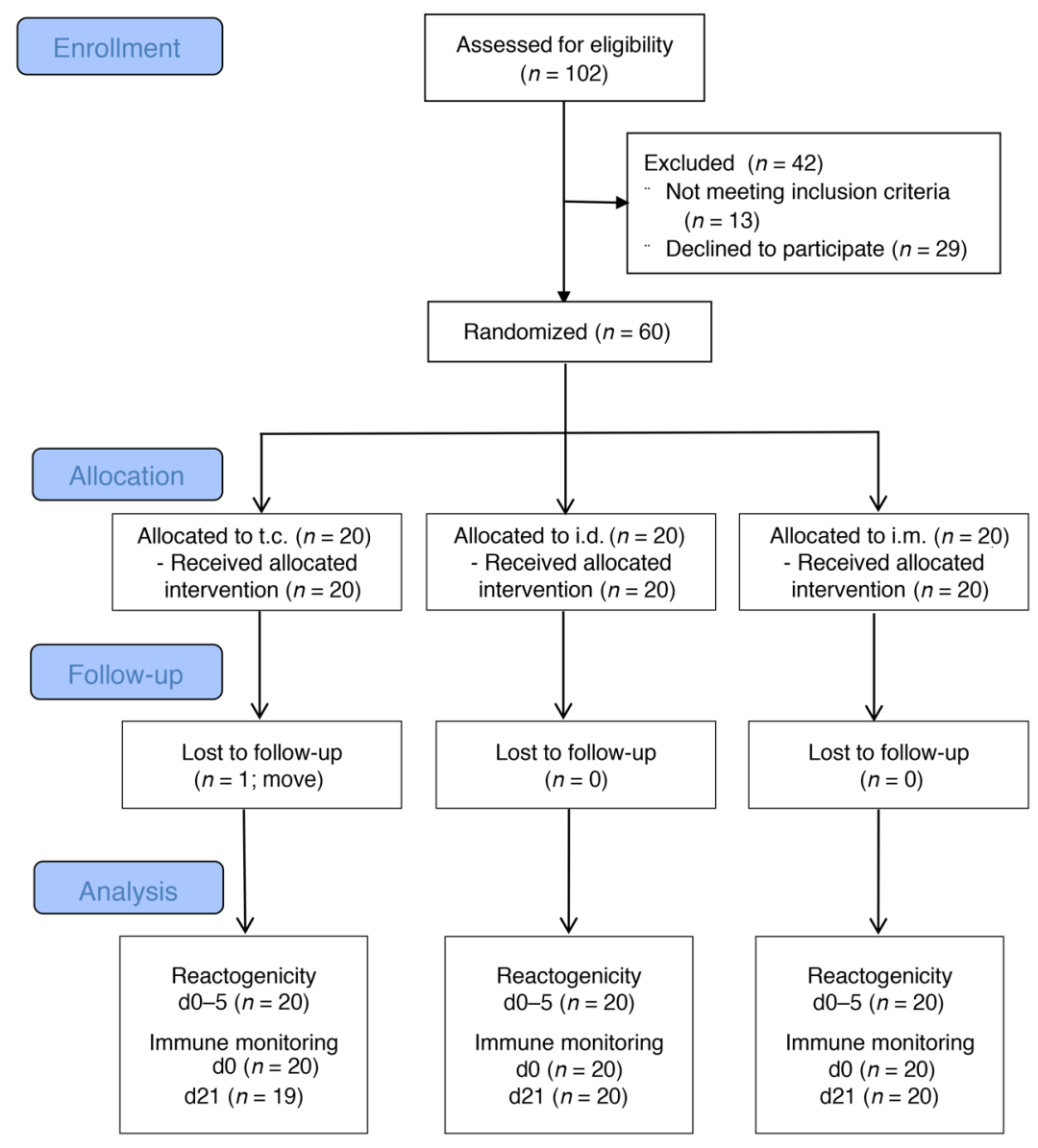

Figure 1. Flow diagram of $\mathbf{6 0}$ participants through the phases of the FLUWAY randomized phase I/II clinical trial. Flow of participants through the FLUWAY clinical trial, according to Consolidated Standard of Reporting Trials (CONSORT). Sixty participants were enrolled, randomized in 3 arms (1:1:1), to receive TIV vaccine by t.c., i.d., or i.m. routes on do. there is also evidence that cytotoxic T lymphocyte (CTL) responses are a surrogate of protection (21, 23). McMichael et al. brought insight to the role of cytotoxic $\mathrm{CD}^{+}$cells with a unique study, showing that in the absence of antibody response to influenza, the presence of CTL activity correlates with reduced viral shedding (23). However, coordinated response between adaptive immune compartments (both humoral and cellular immune responses) is needed to induce an effective immune response and long-term protection after vaccination $(24,25)$. Conventional i.m. immunization fails to induce CD8 cytotoxic responses against seasonal influenza (11).

Recent advances in our understanding of the innate immune system and the use of systems biology approaches are beginning to reveal the fundamental mechanisms by which the innate immune system orchestrates protective immune responses to vaccination (26). Studying the molecular signatures that are induced rapidly after vaccination may help pinpoint biomarkers of immune responses for early identification of impaired responses to vaccines (27). Until now, this mechanistic knowledge has come from studies of vaccine-induced immune regulation in the context of research using systems biology and data integration. Notable studies looked at yellow fever, influenza, pneumococcal, and Ebola vaccination (28-31). These systems vaccinology approaches have identified molecular signatures that correlate with the intensity of protective immune responses. Whether early molecular signatures enable researchers to determine the quality of immune responses and responder and nonresponder status remains to be studied.

Here, we report exploratory results of a randomized phase I/ II clinical trial conducted in 60 healthy subjects $18-45$ years old to compare the immunogenicity of the 2012-2013 seasonal trivalent inactivated influenza vaccine (TIV) administered by the t.c., i.d., and i.m. routes. We propose an integrative approach using systems vaccinology to improve our understanding of the early innate immunity induced by the route of administration and to better study the quality of adaptive immunity, i.e., the antibody and CD8 cellular responses after TIV vaccination.

\section{Results}

Study population. Randomized subjects received A/California/7/2009 (H1N1)pdm09-like strain, A/Victoria/361/2011 (H3N2)-like strain, and B/Wisconsin/1/2010-like strain for the 2012-2013 season by the allocated route of administration on d0 from October 15, 2012, to November 10, 2012, with follow-up until April 25, 2013 (Figure 1). One subject in the t.c. group was lost to follow-up at $\mathrm{d} 21$. Subjects in the 3 groups had similar demographics, and the sex ratio was around $60 \%$ to $40 \%$ women to men in all groups (Supplemental Table 1; supplemental material available online with this article; https://doi.org/10.1172/JCI125372DS1). influenza (19-22). It is generally admitted that humoral response is one correlate of protective response to influenza virus. However, 
A

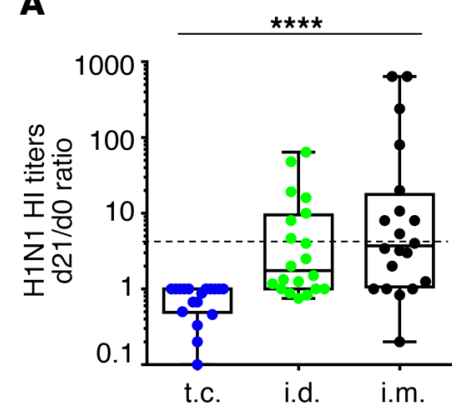

B

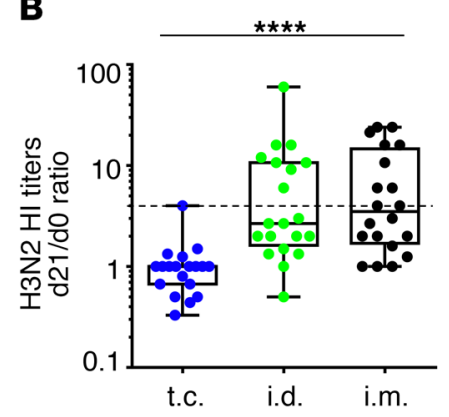

C

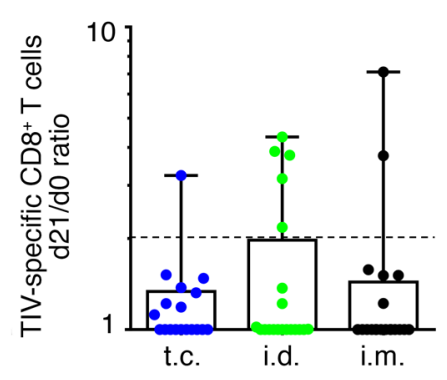

D

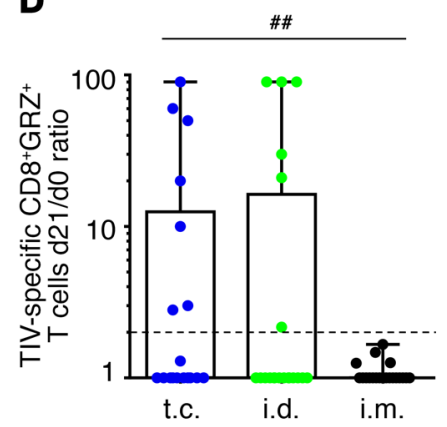

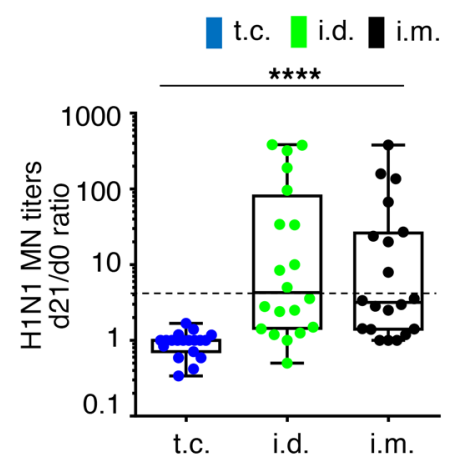
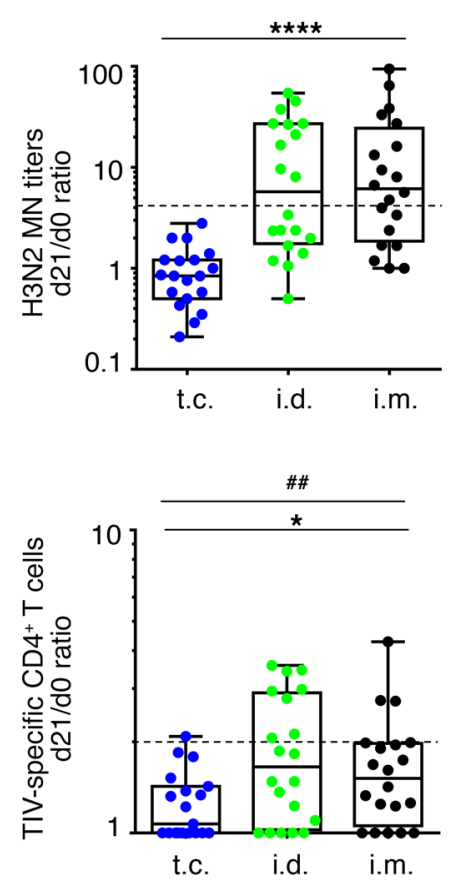

The percentage of subjects who had previously received 1 or more (A)H1N1pdm09 pandemic influenza vaccine $(P=$ $0.0177)$ or seasonal TIV $(P=0.0340)$ injection was significantly higher in the i.d. group $(70 \%$ and $75 \%$, respectively) than in either the t.c. ( $30 \%$ and $35 \%$ ) or the i.m. group (30\% and $40 \%)$. Blood and serum samples were collected from all subjects at visits on do, d1, d21, and month 5 (M5), for the measurement of gene expression at early time points and humoral and cellular responses at d0, d21, and M5. Subjects used diary cards for 5 days after vaccination to report daily local reactions, systemic signs or symptoms, or influenza-like illnesses (ILIs). Neither any ILI nor any serious adverse event was reported during the study. The significantly higher local skin reactogenicity in individuals vaccinated i.d. compared with t.c. and i.m. confirmed previous reports of the safety of these modes of administration (Supplemental Table 2) $(4,10,11,32)$.

Different administration routes induce different qualities of influenza-specific humoral and $T$ cell responses. In an exploratory analysis of our randomized clinical study, a multiparametric analysis was used to define the quality of immune responses after each route of TIV administration. Anti-influenza virus-specific humoral responses were measured by hemagglutinin inhibition (HI) and microneutralization (MN) antibody titers (Supplemental Tables 3 and 4, respectively). TIV-specific $\mathrm{CD} 4^{+}$and $\mathrm{CD} 8^{+}$ $\mathrm{T}$ cells producing cytokines and/or Cd154 and cytotoxic granzyme B (GRZ) were also measured according to the gating strategy given in Supplemental Figure 1. Figure 2 summarizes the ratio at d21 relative to baseline (d0) for each immune parameter. Of note, baseline $\mathrm{HI}$ titers were similar in all subjects, regardless of whether they had or had not received (A)H1N1pdm09 vaccine or seasonal TIV before 2012 (Supplemental Table 1 and Supplemental Figure 2). Moreover, we didn't find any differences in the TIVspecific immune response ratios $(\mathrm{d} 21 / \mathrm{d} 0)$ between individuals who previously received or did not receive at least 1 pandemic H1N1 or seasonal vaccination (Supplemental Figure 3). After TIV administration by the i.d. and i.m. routes, geometric mean titers of $\mathrm{HI}$ antibodies to all $3 \mathrm{influ}-$ enza strains increased significantly (d21 seroprotection rates of $100 \%$ for $\mathrm{A} / \mathrm{H} 3 \mathrm{~N} 2,80 \%$ for $\mathrm{A} / \mathrm{H} 1 \mathrm{~N} 1$, and $35 \%$ $50 \%$ for influenza B; $P \leq 0.0001$ for all 3 viral strains) (Supplemental Table 3). As expected, t.c. vaccination with TIV did not induce higher HI or MN (Figure 2, A and B) antibody titers against $\mathrm{A} / \mathrm{H} 1 \mathrm{~N} 1$ and $\mathrm{A} / \mathrm{H} 3 \mathrm{~N} 2$ strains than did i.d. and i.m. vaccination (11). Intensity of immune responses at d0 and d21 are shown in Supplemental Figure 4.

We monitored TIV-specific $\mathrm{CD} 4^{+}$and $\mathrm{CD} 8^{+} \mathrm{T}$ cells expressing intracellular IL-2, IFN- $\gamma$, TNF- $\alpha$, and/or cellsurface Cd154 (CD40 ligand) as well as TIV-specific CD8 ${ }^{+}$ GRZ $^{+} \mathrm{T}$ cells (Figure 2, C and D). The d21/d0 ratios of the TIV-specific cytokine-producing $\mathrm{CD} 8^{+} \mathrm{T}$ cells were similar in all 3 arms and highly heterogeneous between individuals (Figure 2C) (Kruskal-Wallis test; $P$ not significant). We found significant differences $(P=0.0369)$ among the 3 arms for TIV-specific cytokine-producing $\mathrm{CD} 4^{+} \mathrm{T}$ cells. 
This was confirmed by a $\chi^{2}$ test applied to responders and nonresponders as defined by a d21/d0 ratio of at least 2 for vaccine-specific $\mathrm{CD} 4^{+} \mathrm{T}$ cells. We found significant differences $(P=0.0204)$ among the 3 routes; response rates were higher for i.d. $(8 / 20)$ than i.m. $(3 / 20)$ or t.c. $(1 / 19)$.

Interestingly, the $\mathrm{d} 21 / \mathrm{dO}$ ratios of the TIV-specific $\mathrm{CD}^{+} \mathrm{GRZ}^{+}$ $\mathrm{T}$ cells were similar in all 3 arms according to a Kruskal-Wallis test, although after application of the $\chi^{2}$ test to responders and nonresponders we found a significantly higher response rate observed in the t.c. (7/18) and i.d. (6/20) groups compared with the i.m. group $(0 / 20)(P=0.0098$; Figure 2D). Overall, the intensity of vaccinespecific humoral, cytokine-producing $\mathrm{T}$ cell and $\mathrm{CD} 8^{+} \mathrm{GRZ}^{+} \mathrm{T}$ cell responses differed by administration route, with higher humoral responses ( $\mathrm{HI}$ and $\mathrm{MN}$ antibody titers) after i.m. and i.d vaccination and higher vaccine-specific $\mathrm{CD}^{+} \mathrm{GRZ}^{+} \mathrm{T}$ cells after t.c. and i.d. vaccination.

Early innate signatures discriminate immunological outcome. We used transcriptomic approaches to compare the quality of early innate immunity regardless of adaptive immunity outcomes. Comparison of the expression profiles of blood genes at 24 hours after vaccination (d1) and at baseline (d0) for each route of immunization showed 389 genes differentially expressed in the i.m. arm, 127 in the i.d. arm, and 4 in the t.c. arm (significance for the Bayesian $t$ test was set at a corrected $P<0.1$ ), as represented in a Venn diagram (Figure 3A). We noticed that 24 genes are common between the routes. Of note, comparison of gene expression at do (baseline) of all samples showed no significant difference among study arms (Supplemental Figure 5).

To study whether these 496 genes could differentiate immunization routes, we performed principal component analysis (PCA) of the data for the 496 genes from each individual (Figure 3B) and a hierarchical clustering (HCL) of the individuals (Figure 3C) (t.c., blue; i.d., green; and i.m., black). As the HCL analysis shows (Figure 3C), the samples were distributed into 2 major clusters: C1 (gray) and C2 (red). Of note, samples from individuals are distributed in both clusters. C1 includes $2 / 17,16 / 18$, and 10/17 t.c.-, i.d.-, and i.m.-vaccinated individuals, respectively. C2 includes the other 15/17, 2/18, and 7/17 t.c-, i.d.-, and i.m.vaccinated individuals, respectively. Using Ingenuity pathway analysis (IPA) on 496 genes, we found that this C1-C2 dichotomy is due mainly to the genes involved in multiple pathways, such as those for antigen-presentation (IPA: $P=1.32 \times 10^{-5}$ ), DC maturation (IPA: $P=7.14 \times 10^{-4}$ ), and IFN signaling (IPA: $P=9.18 \times 10^{-15}$ ). Figure 3D displays the top pathway IFN signaling, which includes STAT1, IRF9, IFI35, IRF7, PSMB8, and FCGR1A from a cluster of significantly upregulated genes in C1 compared with C2, among the 496 genes. It has been previously shown that CXCL10 is also one of the key mediators of immune response (33). We thus measured CXCL10 production in subjects' serum samples at $\mathrm{d} 1$ compared with $\mathrm{d} 0$. We found that $\mathrm{d} 1 / \mathrm{d} 0$ ratios of CXCL10 levels were significantly higher in the $\mathrm{C} 1$ than in the $\mathrm{C} 2$ cluster $(P<0.0001)$ (Figure 3D), CXCL10 serum levels being correlated with influenza-specific MN antibody titers (strains H1N1: $P=$ 0.0002, $r=0.49$; and H3N2: $P<0.0001, r=0.61$ ).

Based on the 496 genes differentially expressed at $\mathrm{d} 1$ and the distribution of samples according to the HCL, we analyzed adaptive immunity (d21) in C1 and C2 clusters. We observed significant differ- ences in the immune responses (d21/d0 ratio) between $\mathrm{C} 1$ and $\mathrm{C} 2$ (Figure 3, E and F). C1 individuals had significantly higher influenzaspecific MN antibody titers (H1N1: $P=0.0013$, Figure 3E; and H3N2: $P=0.0004$, Supplemental Figure 6A) than C2 subjects. Similar data were observed for $\mathrm{HI} \mathrm{Ab}$ titers in $\mathrm{C} 1$ and $\mathrm{C} 2$ (data not shown). In contrast, TIV-specific $\mathrm{CD}^{+} \mathrm{GRZ}^{+} \mathrm{T}$ cell responders were more frequent in the $\mathrm{C} 2$ than the $\mathrm{C} 1$ group $(P=0.0406)$ (Figure $3 \mathrm{~F}$ ). However, we did not observe any significant difference in distribution of TIVspecific $\mathrm{CD}^{+}$and $\mathrm{CD} 8^{+} \mathrm{T}$ cells expressing intracellular IL-2, IFN- $\gamma$, TNF- $\alpha$, and/or cell-surface Cd154 (CD40 ligand) in C1 and C2 (Supplemental Figure 6, B and C). This was confirmed by the immunological assays performed at $\mathrm{d} 0$ and $\mathrm{d} 21$, according to $\mathrm{C} 1$ and $\mathrm{C} 2$ sample clusters (Supplemental Figure 7). In conclusion, the expression profile of the whole-blood genes (d1/d0) allowed us to distinguish H1N1- and H3N2-specific antibody responses from TIV-specific $\mathrm{CD}^{+} \mathrm{GRZ}^{+} \mathrm{T}$ cell responses.

Early innate gene signature and serum levels of cytokines allows identification of humoral and cytotoxic $T$ cell responders. Among the 496 differentially expressed genes (d1/d0), 80 were correlated with $\mathrm{MN}$ antibody titers $(P<0.05,0.3$, less than $r<0.6)$ mainly involved in interferon signaling (IPA: $P=9.18 \times 10^{-5}$ ) and antigen presentation pathways (IPA: $P=4.11 \times 10^{-4}$ ) and 31 genes with TIV-specific CD8 ${ }^{+} \mathrm{GRZ}$ T cell responses $(P<0.05$, 0.3 , less than $r<0.4$ ) involved in metabolic pathways (IPA: $\left.P=5.88 \times 10^{-3}\right)$. We then looked at whether these genes were differentially expressed in responders and nonresponders, as defined in Figure 2. The top 5 genes with significantly different gene expression levels between humoral responders and nonresponders ( $\mathrm{MN}$ antibody titers) are presented in Figure 4A and Supplemental Table 5, as are the top 4 genes with significantly different gene expression levels among subjects with and without TIV-specific CD8 ${ }^{+} \mathrm{GRZ}^{+} \mathrm{T}$ cell responses.

Hierarchical clustering based on the expression profile of these 9 genes revealed 2 clusters of subjects: S1 (purple) including $3 / 17$ (9\%) vaccinated by the t.c. route, $13 / 18$ (39\%) by i.d., and $17 / 17$ (52\%) by i.m., and S2 (yellow), which included 14/17 (74\%) vaccinated by t.c. and 5/18 (26\%) by i.d. (Figure $4 \mathrm{~B}$ ). We observed significantly higher influenza-specific $\mathrm{MN}$ antibody responses in S1 than in S2 (A/H3N2: $P=0.0002 ; \mathrm{A} / \mathrm{H} 1 \mathrm{~N} 1: P=0.0005)$ and the same distribution is observed for the $\mathrm{d} 1 / \mathrm{d} 0$ ratios of CXCL10 concentrations (Figure 4, C and D and Supplemental Figure 8). In contrast, the $\mathrm{S} 2$ cluster contains significantly higher numbers of individuals with higher TIV-specific $\mathrm{CD}^{+} \mathrm{GRZ}^{+} \mathrm{T}$ lymphocyte counts $(P=0.0007)$ (Figure $4 \mathrm{E})$. This is confirmed by the immunological assays at $\mathrm{d} 0$ and $\mathrm{d} 21$ independently according to S1 and S2 samples clusters (Supplemental Figure 9). Moreover, it has been shown that i.d. vaccination induces both TIV-specific $\mathrm{MN}$ titers and $\mathrm{CD} 8^{+} \mathrm{GRZ} \mathrm{Z}^{+} \mathrm{T}$ cell responses, whereas t.c. vaccination preferentially induces $\mathrm{CD}^{+} \mathrm{GRZ}^{+} \mathrm{T}$ cell responses. However, we did not observe any significant difference in distribution of TIV-specific $\mathrm{CD}^{+}$and $\mathrm{CD} 8^{+} \mathrm{T}$ cells expressing intracellular IL-2, IFN- $\gamma$, TNF- $\alpha$, and/or cell-surface Cd154 (CD40 ligand) in S1 and S2 (Supplemental Figure 8, A and B).

PCA performed on 9 genes (genes correlated with immune responses and were differentially expressed between responders and nonresponders) showed sample distribution according to the administration route (t.c., blue; i.d., green; i.m., black; Figure 5A). 
A

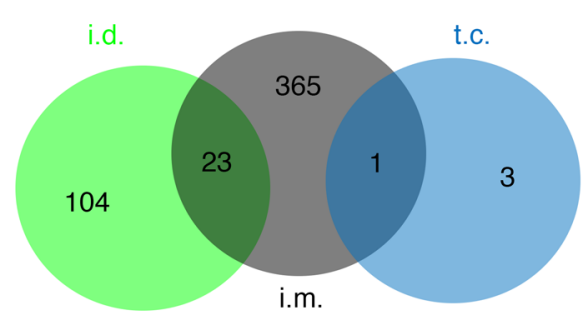

C

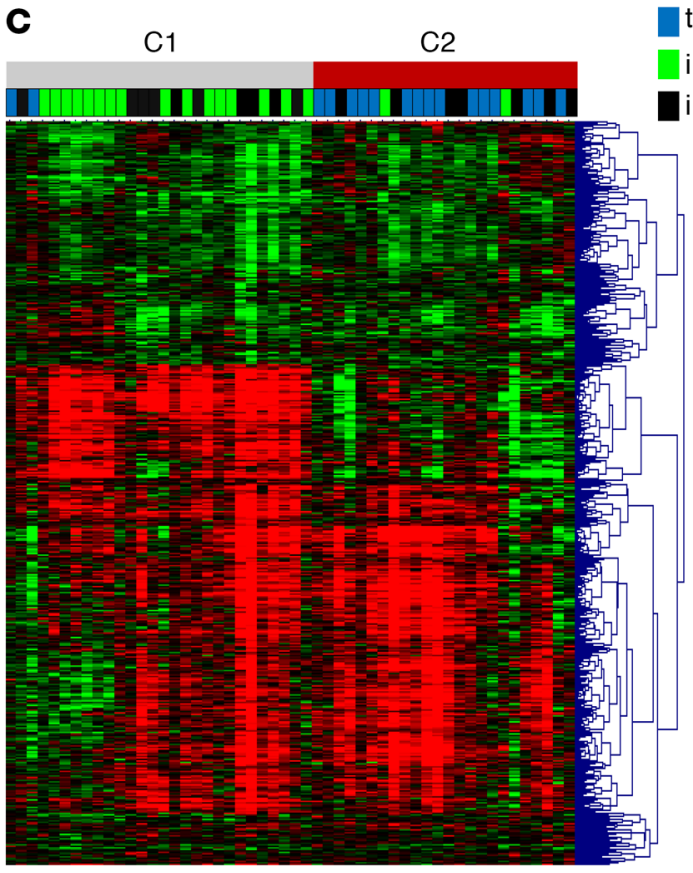

Fold-change $\quad 1.0$ $\mathrm{d} 1 / \mathrm{d} 0$

E

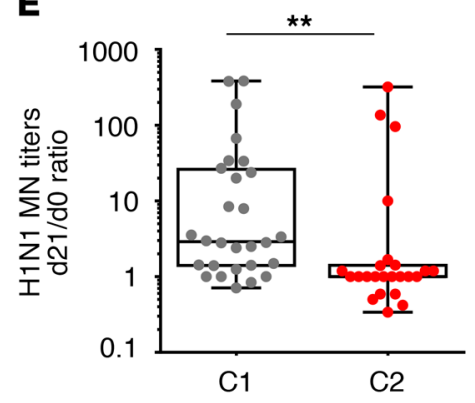

$\mathbf{F}$

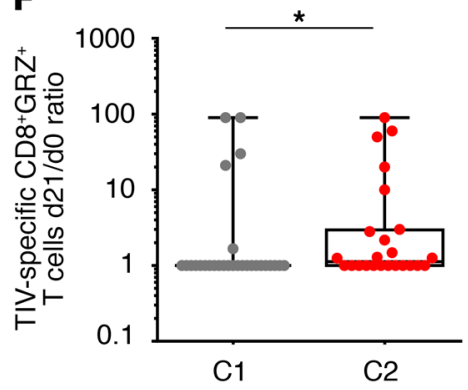

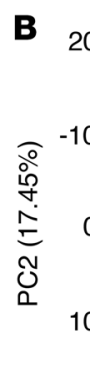

$-20$

$-20$

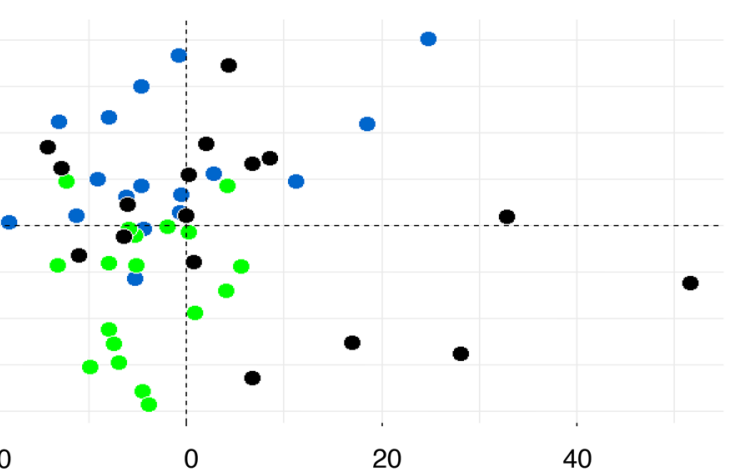

PC1 (32.72\%)

\section{D}

t.c. D
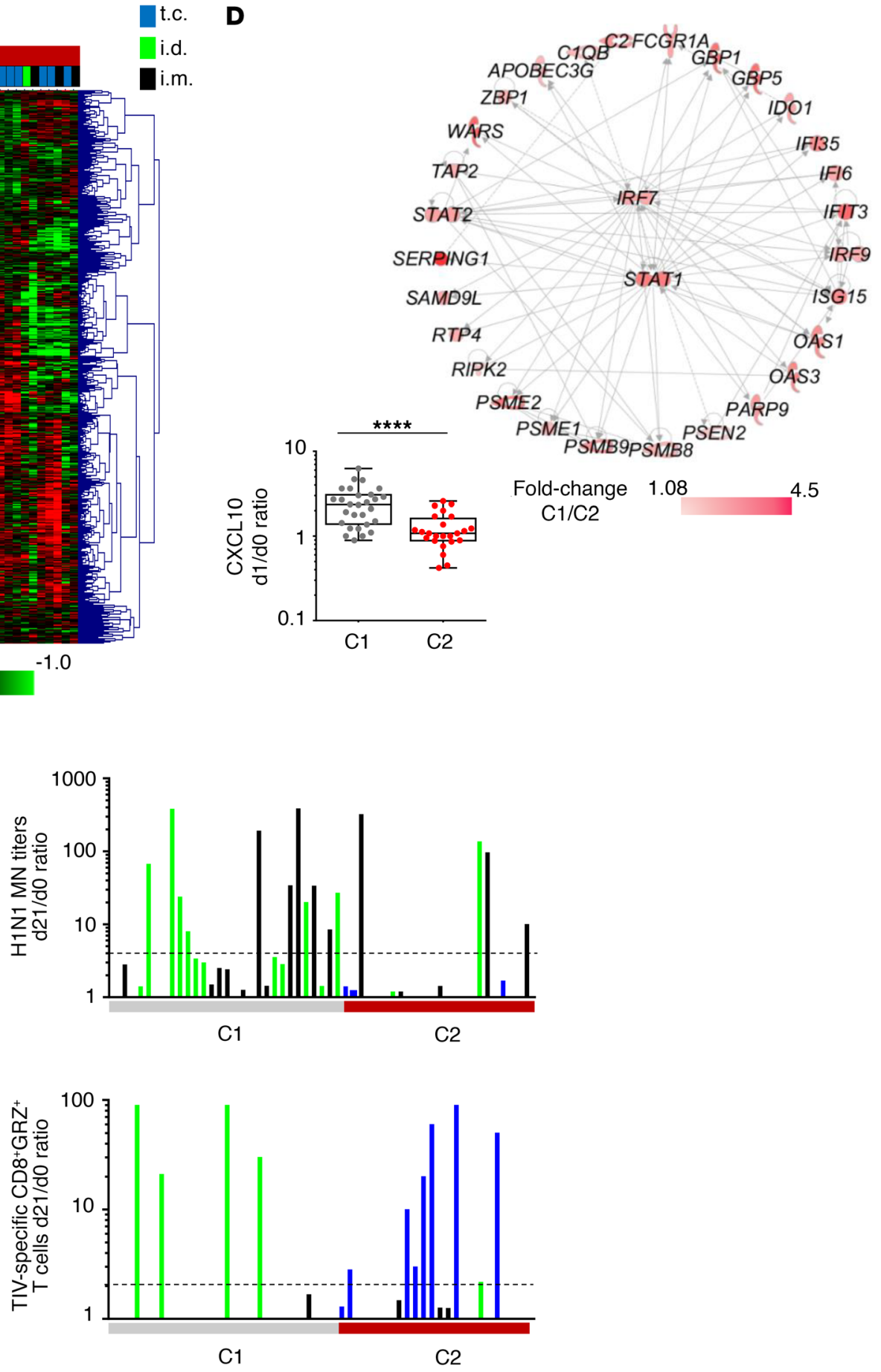
Figure 3. Early innate signature discriminates immunological outcome, rather than route of immunization. (A) Venn diagram of the genes differentially expressed between paired $\mathrm{d} 1$ and $\mathrm{d} 0$ for t.c. (blue, $n=17$ ), i.d. (green, $n=18$ ), and i.m. (black, $n=17$ ). Significance for the Bayesian $t$ test was set at a corrected $P$ less than 0.1. The number of genes differentially expressed between $\mathrm{d} 1$ and $\mathrm{d} 0$ is indicated for each arm. (B) PCA of these 496 genes differentially expressed between $\mathrm{d} 1$ and $\mathrm{do}$. Vaccine administration routes are indicated. (C) Gene and sample hierarchical clustering is based on the expression profiles of these 496 genes. Two sample clusters named $\mathrm{C} 1$ (gray) and $\mathrm{C} 2$ (red) are observed. For $\mathrm{C} 1, n=28$ : t.c. $n=2$, i.d. $n=16$, i.m. $n=10$. For $C 2, n=24$ : t.c. $n=15$, i.d. $n=2$, i.m. $n=7$. The color-gradient from green $(-1$, low) to red $(1$, high) indicates fold change $\mathrm{d} 1 / \mathrm{d} 0$ gene expression $\left(\log ^{2}\right)$. (D) A major cluster of gene expression, with expression significantly different between $\mathrm{C} 1$ and $\mathrm{C2}$, corresponds to the IFN-signaling pathway. Of note, IPA analysis indicated master genes (IRF7 and STAT1) at the center of the network. The red gradient indicates the differential expression between $\mathrm{C} 1$ and $\mathrm{C2}$ (1.08- to 4.5-fold change gene expression $\mathrm{C} 1 / \mathrm{C} 2$ ). And the box-and-whisker plot (from the bottom up: the minimum 25th percentile Q1, median, 75th percentile Q3, and maximum values) with minimum to maximum showing all sample points ( $y$ axis of graph in $\log ^{10}$ scale). It represents the d1/d0 ratio of the CXCL10 serum level from the C1 (gray) and C2 (red) samples. Box-and-whisker plots represent (E) H1N1-specific MN antibody titers (d21/d0 ratios) and (F) TIV-specific CD8 ${ }^{+} \mathrm{CRZ}^{+} \mathrm{T}$ cells (d21/d0 ratios) according to $\mathrm{C} 1$ and $\mathrm{C} 2$ clusters (left panels). Respective histograms in $\mathbf{E}$ and $\mathbf{F}$ show immune response intensities for each individual in $\mathrm{C} 1$ (gray) and $\mathrm{C} 2$ (red) clusters (right panels). The Mann-Whitney $t$ test compares $\mathrm{C} 1$ and $\mathrm{C} 2$ for these responses; ${ }^{*} P<0.05$; ${ }^{*} P<0.01$; ${ }^{* * *} P<0.0001$.

We then used logistic regression analysis to consider whether this minimal gene signature, together with the CXCL10 serum levels, might supposed response by either TIV-specific $\mathrm{CD}^{+} \mathrm{GRZ}^{+}$ $\mathrm{T}$ lymphocytes or $\mathrm{MN}$ antibody titers. The receiver operating characteristics (ROC) curve showed that the 9 genes and the $\mathrm{d} 1$ / dO ratios for CXCL10 serum levels were the most accurate prognosticators of responses by TIV-specific $\mathrm{CD}^{+} \mathrm{GRZ}^{+} \mathrm{T}$ cells (AUC: 0.9354) and by H1N1- and H3N2-specific MN antibody titers (AUC: 0.9676 and 0.9585, respectively) (Figure 5B). Figure 5C shows the number and percentage of individuals correctly foreseen to be responders by their status for each immune parameter. The percentage of individuals predicted as responders for H1N1and $\mathrm{H} 3 \mathrm{~N} 2$-specific $\mathrm{MN}$ antibody and TIV-specific $\mathrm{CD}^{+} \mathrm{GRZ}^{+} \mathrm{T}$ cell responses is $100 \%, 84.21 \%$, and $83.33 \%$, respectively.

\section{Discussion}

We believe this work is the first phase I/II clinical trial that aims to assess the safety and immunogenicity of TIV administered by t.c., i.d., and i.m. routes, and provides important insights into the dichotomy shown by early innate markers for the induction of either TIV-specific humoral or CD8 responses.

We found that TIV immunogenicity varied according to delivery routes. Stronger humoral responses (HI and MN antibody titers) were observed with i.m. and i.d. vaccination compared with t.c. vaccination, and vaccine-specific cytotoxic $C D 8^{+} \mathrm{GRZ}^{+} \mathrm{T}$ lymphocyte responses were higher after t.c. and i.d. vaccination, compared with i.m. vaccination. These results confirm our previous demonstration that a t.c. route, preferentially targeting LCs, induces $\mathrm{CD}^{+} \mathrm{T}$ cell responses $(6,7,11,13)$. It also confirms that the i.d. vaccination route induces both humoral and $\mathrm{CD} 8^{+} \mathrm{T}$ cell responses due to the presence of LC and $\mathrm{dDC}$ populations. These cells include
LCs in the epidermis and dDCs in the dermal layer, both involved in processing and presenting pathogen-derived antigens to $\mathrm{T}$ and $\mathrm{B}$ cells from draining lymph nodes to initiate the antigen-specific cellular and humoral responses. Influenza vaccine by the i.d. route (a) elicits stronger immune responses in preclinical studies than those induced by the i.m. route (34-36), (b) may improve response in high-risk populations such as the elderly (37-40), and (c) requires a lower vaccine dose than the i.m. route $(17,41)$. We proposed that immunization routes dictate the immunological outcomes of either humoral or $\mathrm{CD}^{+}$cellular responses in mice and humans $(6,11)$. In our study, we also took into account the impact of previous vaccination status on innate and adaptive immunity. We did not find any significant difference at baseline in both transcriptomic profile and adaptive immunity in previously vaccinated individuals compared with individuals who were not vaccinated in previous years.

We analyzed whole-blood gene expression at $\mathrm{d} 1$ relative to do. Although previous work by others has studied later time points (D3-D7) $(29,33)$, we chose our design to study early innate immunity only with samples collected at baseline and d1 after vaccination. Because the route of immunization dictates innate immune response, we expected approximately 3 gene expression clusters representing the 3 modes of administration. Surprisingly, however, we observed that subjects were distributed into 2 major clusters (C1 and C2). This dichotomy was mainly due to the IFN-signaling pathway and CXCL10 production in serum 1 day after vaccination. C1 and C2 each displayed 1 of the 2 major immunological outcomes: humoral (influenza-specific MN antibodies) and $\mathrm{CD}^{+} \mathrm{GRZ}^{+} \mathrm{T}$ cells, respectively, at $\mathrm{d} 21$ relative to do. Analysis of $\mathrm{HI}$ antibody titers produced similar results (data not shown). Overexpression of the IFN-signaling pathway (at D3 after vaccination) has previously been correlated with a higher TIVspecific HI antibody response $(28,33)$. Similarly, we found that the IFN-signaling pathway (at d1) and the CXCL10 serum level characterized individuals with high humoral responses at d21. Other studies have identified this chemokine, known to mediate antiviral immunity, in serum 3 days after influenza immunization by the i.m. route (where it was negatively correlated with $\mathrm{HI}$ antibody response 28 days afterward) (33) and 3 days after recombinant Vesicular Stomatitis Virus-Zaire Ebola virus (rVSV-ZEBOV) vaccination administration, where it was finally found to be an independent correlate of antibody response (31). On the other hand, early upregulation of IFN-signaling and antigen-presentation pathways after i.m. TIV immunization is known to be associated with higher influenza-specific HI antibody response $(28,33)$ and to result in overexpression of STAT1, IRF9, IFI35, IRF7, PSMB8, and FCGR1A, as found in our analysis for both the i.d. and i.m. routes.

Systems vaccinology is helping to increase knowledge about the molecular networks that orchestrate immunity in response to vaccination in humans $(42,43)$ and to identify signatures used to predict vaccine-induced immunity, such as humoral and cellular responses $(44,45)$. Studies of the innate blood transcriptome have helped to predict the magnitude of later antibody and $\mathrm{CD}^{+} \mathrm{T}$ cell responses against the live-attenuated yellow fever vaccine $(30,43,46)$. Predicting $\mathrm{CD}^{+} \mathrm{T}$ cell responses against influenza vaccines is more challenging because of the poor induction of these immune responses by i.m. immunization. In circumstances when humoral immunity is not protective, or 
A
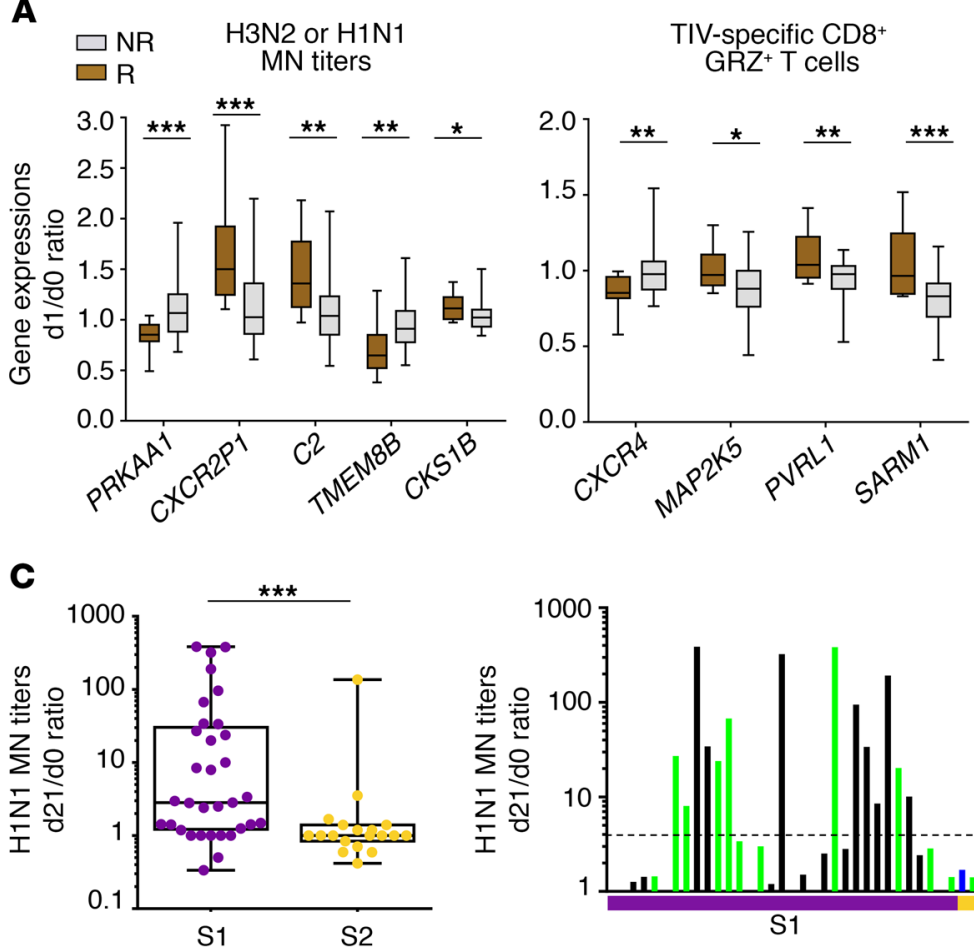

B
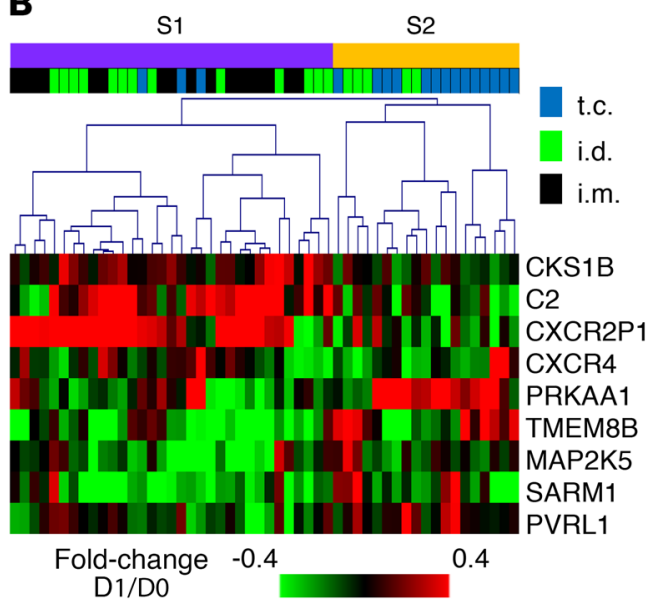

D
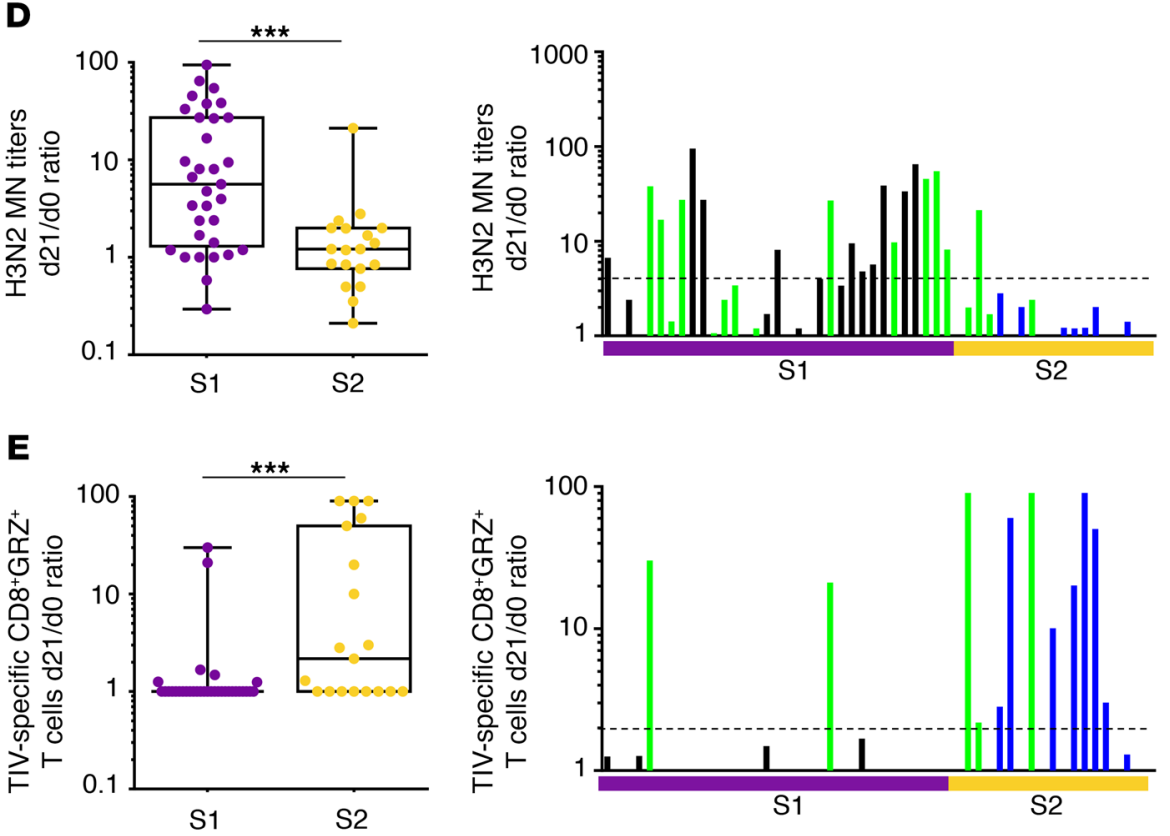

Figure 4. Minimal innate signature correlates with immunological outcome independently of route of immunization. (A) Box-and-whisker plots (minimum to maximum) are depicted for 9 gene expression ratios between $\mathrm{d} 1$ and $\mathrm{d} 0$ in nonresponders (NR; gray) and responders (R; brown) (d21/d0 ratio $\geq$ 4 for A/H3N2 or A/H1N1 MN titers [left panel] and ratio $\geq 2$ for TIV-specific CD8 ${ }^{+} G R Z^{+} T$ cell responses [right panel]). The Mann-Whitney $t$ test compares responders and nonresponders $\left({ }^{*} P<0.05 ;{ }^{* *} P<0.01 ;{ }^{* *} P<0.001\right)$. (B) Gene and sample hierarchical clustering of 9 gene expression profiles revealed 2 clusters of subjects: S1 (purple, $n=33$ ), with $3 / 17$ t.c., $13 / 18$ i.d., and all i.m. subjects ( $n=17 / 17$ ), and S2 (yellow, $n=19$ ), with $14 / 17$ t.c. and $5 / 18$ i.d. subjects. Samples are color-coded according to vaccination route: t.c. (blue), i.d. (green), and i.m. (black). The color gradient from green (-0.4, low) to red (0.4, high) indicates the intensity of gene expression. Box-and-whisker plots with the minimum to maximum points of d21/d0 immune responses (from the bottom up: the minimum 25th percentile Q1, median, 75th percentile Q3, and maximum values) for (C) A/H1N1 MN titers, (D) A/H3N2 MN titers, and (E) TIVspecific CD8+CRZ ${ }^{+} \mathrm{T}$ cell responses in the S1 (purple) and S2 (yellow) clusters (left panels). The Mann-Whitney $t$ test compared the S1 and S2 clusters $\left({ }^{* *} P<0.001\right)$. Respective histograms show immune response intensities for each individual in the S1 (purple) and S2 (yellow) clusters (right panels). t.c., blue; i.d., green; and i.m., black. 
A
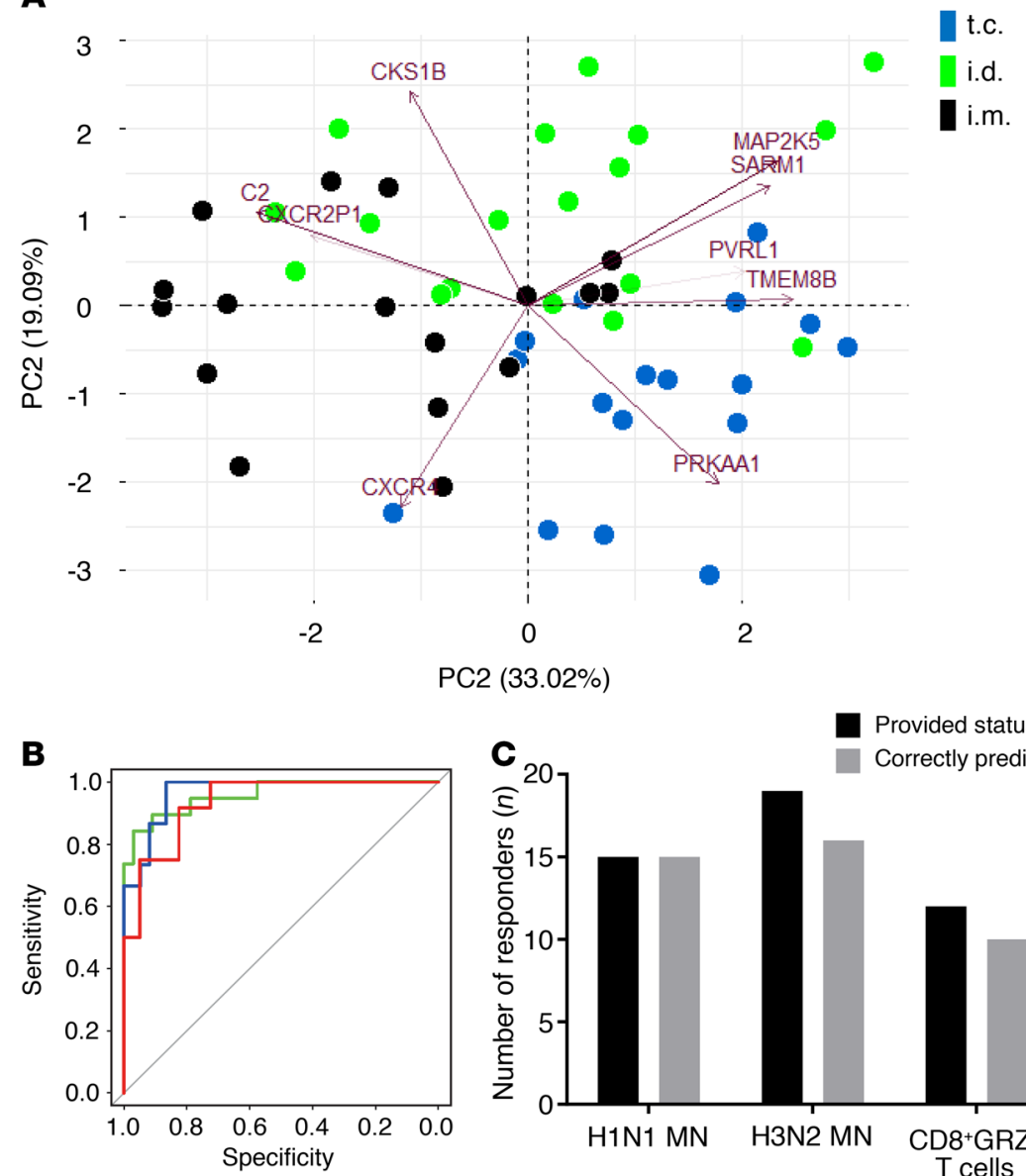

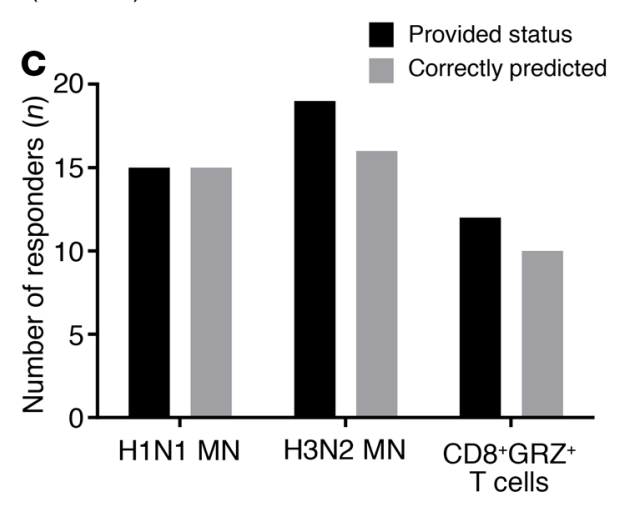

Figure 5. Minimal innate signature and serum level of CXCL10 allows identification of humoral and cytotoxic $T$ cell responders after TIV vaccination by different routes of administration. (A) Principal component analysis of the 9 gene expression profiles (d1/d0). Sample vaccine administration routes are color coded: i.d. in green $(n=18)$, i.m. in black $(n=17)$, and t.c. in blue $(n=$ 17). (B) ROC curves showing the specificity and sensitivity of the logistic regression models, i.e., the proportion of correctly anticipated responders and nonresponders, respectively. The curves correspond to the $\mathrm{d} 21 / \mathrm{d} 0$ ratio of immune responses: H1N1-specific MN antibody titers (blue; AUC: 0.9676), H3N2-specific MN antibody titers (green; AUC: 0.9585), and TIV-specific CD8 ${ }^{+} \mathrm{CRZ}^{+} \mathrm{T}$ cells (red; AUC: 0.9354). The 9 gene expression profiles and the CXCL10 serum levels significantly explain each of these variables. (C) The histogram represents the number of responders for each immune response: H1N1/ H3N2 MN antibodies (d21/d0 ratio $\geq 4$ ) and TIV-specific CD8 ${ }^{+} \mathrm{CRZ}^{+} \mathrm{T}$ lymphocytes (d21/d0 ratio $\geq 2$ ), in regard to provided status (black) and correctly predicted status (gray) based on the early expression of the 9 genes and on CXCL10 serum levels (d1/d0). is inadequately protective, against the influenza strain causing an outbreak, cytotoxic $\mathrm{CD} 8^{+} \mathrm{T}$ cells might provide some degree of protection against infection and attenuate disease severity and mortality (21). This is especially the case for the majority of $\mathrm{CD} 8^{+} \mathrm{T}$ lymphocytes directed to epitopes that are cross-reactive between viral subtypes $(47,48)$. The t.c. route of immunization gave us the opportunity to investigate the induction of $\mathrm{CD} 8^{+}$ responses without humoral responses. Among the 496 genes differentially expressed (on $\mathrm{d} 1$ compared with $\mathrm{d} 0$ ), we found 5 genes correlated with $\mathrm{A} / \mathrm{H} 1 \mathrm{~N} 1$ and $\mathrm{A} / \mathrm{H} 3 \mathrm{~N} 2 \mathrm{MN}$ antibody titers and 4 with TIV-specific $\mathrm{CD} 8^{+} \mathrm{GRZ}^{+} \mathrm{T}$ cell responses. They constitute so-called minimal gene signature. We found an innate gene signature induced 1 day after TIV vaccination that distinguished patients with late MN antibody responses from those with $\mathrm{CD} 8^{+} \mathrm{GRZ}^{+} \mathrm{T}$ lymphocyte responses, regardless of delivery route. In our study, CXCR2P1, C2, and CKS1B, induced on d1 early after TIV immunization, were positively correlated with A/H1N1 MN response at d21, whereas PRKAA1 and TMEM8B were negatively correlated with it. Among the genes correlated to $\mathrm{A} / \mathrm{H} 1 \mathrm{~N} 1 \mathrm{MN}$ antibodies, CKS1B is involved in cell cycle and proliferation, JAK/STAT3 signaling (through its role in cytokine receptor signaling and inflammation), and the MEK/ERK pathway (where CKS1B regulates monocyte autophagy) (49). C2 is involved in the complement system; it triggers inflammation and plays a central role in promoting humoral immune response through antigen uptake and presentation to $\mathrm{B}$ and $\mathrm{T}$ cells (50). CXCR2P1 is the pseudogene of the IL-8 receptor and is mainly involved in neutrophil migration (51). Protein kinase PRKAA1, the catalytic subunit of AMPK, is expressed principally in macrophages and lymphocytes and plays a pivotal role in inducing proinflammatory signals through TAK1 and NFkB activation (52). AMPK promotes inflammation, innate immunity, and antiviral defenses through modulation of the host protein, a stimulator of $I F N$ gene signaling (STING) (53). TMEM8B is involved in cell signal transduction and controls cell-cycle progression (54).

Our study also identified the following gene signature related to $\mathrm{CD} 8{ }^{+} \mathrm{GRZ}^{+} \mathrm{T}$ cell response: MAP2K5, PVRL1, SARM1, and CXCR4. Interestingly, MAP2K5 is a cofactor required for innate immune responses dependent on TLR7 and TLR9, which are tolllike receptors of DCs, macrophages, and natural killer cells (55). CXCR4 is a chemokine receptor involved in migration and proliferation of white blood cells and plays a chemotactic role for neutrophils, monocytes, and lymphocytes $(56,57)$. Of note, PVRL1 is involved in cell movement and proliferation and is expressed on DCs. SARM1 is a TLR adaptor carrying a TIR (toll interleukin receptor) domain to ensure efficient immune responses against bacterial infections by negatively regulating TLR signaling (58). Another study has also proposed biomarkers related to apoptotic pathways that reflect impaired TIV responses in young compared with older individuals; however, these biomarkers are related to the individuals' chronological ages (59). 
Franco et al. identified 20 genes exhibiting a transcriptional response to vaccination, and significant genotype effects on gene expression, which are correlated to the intensity of antibody responses (60). A number of loci that have the strongest evidence of genetic variation might also influence the intensity of humoral response to vaccine. These results suggest that genetic variation in identified genes could also influence immunological outcomes.

Several inflammatory cytokines were measured in the sera at d1. We found slightly but nonetheless significantly more serum level of IL-6 production following vaccination (defined by a d1/ d0 ratio $\geq 2)$ in S2 $(7 / 19)$ compared with S1 samples (4/33) $\left(\chi^{2}\right.$, $P=0.0356$ ) (Supplemental Figure 10). Of note, the adjusted ROC curve showed that the 9 genes and the $\mathrm{d} 1 / \mathrm{d} 0$ ratios for IL- 6 and CXCL10 serum levels were the most accurate predictors of responses by TIV-specific $\mathrm{CD}^{+} \mathrm{GRZ}^{+} \mathrm{T}$ cells (AUC: 0.9417 , predicted responders 91.66\%) (Supplemental Figure 10). IL-6 production by DCs enhances CD ${ }^{+} \mathrm{T}$ cell proliferation, triggers TCR activation, and is dispensable for memory $\mathrm{CD}^{+} \mathrm{T}$ cell generation in mice (61). In addition, the interaction of keratinocytes, LCs, and dDCs after cutaneous vaccination tends to promote inflammatory cytokine production, such as IL1- $\alpha$, IL-6, IL-8, and CXCL10, that might contribute to the overall inflammatory reaction and further promote the attraction of inflammatory cells at the immunization site $(62,63)$. Moreover, vaccine adjuvant monophosphoryl lipid A (MPLA) enables effective $\mathrm{CD} 8^{+} \mathrm{T}$ cell differentiation into cytotoxic cells in the presence of DC-induced IL-6. MPLA and alum with an epitope from a nucleoprotein highly conserved between influenza strains generate cytotoxic $\mathrm{T}$ lymphocyte differentiation and long-lived memory $\mathrm{T}$ cells that protect mice against influenza A (64). Additional studies are necessary to determine whether the genes identified have a causal relationship to either the antibody response or the cell-mediated immune response to the vaccine.

In conclusion, we identified potential blood biomarkers at the level of gene expression and CXCL10 in serum that are good indicators of either humoral or cytotoxic $\mathrm{T}$ cell response after seasonal influenza vaccination. It is clear that further studies and validation in clinical trials are necessary to identify both a larger group of biomarkers induced after vaccination and predictors of the quality of immune responses. These studies must consider the variability, sensitivity, and specificity of these biomarkers in humans. Unfortunately, we did not have access to such studies comparing routes of immunization since our proposed work is the only clinical trial comparing 3 routes of administration. Although the transformation of data into predictive markers of immunity remains a challenge (65, 66), this study provides what we believe is new insight into the impact of the route of immunization and innate signature in the quality of immune responses.

\section{Methods}

Clinical study protocol. The FLUWAY (Routes of Immunization and Flu Immune Responses) randomized phase I/II clinical study enrolled 60 volunteers aged $18-45$ years from October 15,2012 , to November 10 , 2012, with follow-up until April 25, 2013; 20 were allocated to each vaccine administration group in a 1:1:1 ratio after randomization at the Cochin-Pasteur Center for Clinical Investigation in Vaccinology at Cochin Hospital, Paris, France. The study has been registered at
ClinicalTrials.gov (NCT01707602). Exclusion criteria were as follows: pregnancy (positive urine test); HIV, hepatitis B, or hepatitis C virus infections; Guillain Barré syndrome; immunosuppressive treatment or other immunodeficiency; allergy to a vaccine component; medical history of skin cancer; acute infection or vaccination within 4 weeks of enrollment; body mass index lower than 21 or greater than 26; skin phototype V-VI; excessive terminal hair growth; and planned sun exposure 6 weeks before or during the study.

The number of subjects to be included in the research is not based on a statistical hypothesis but on the feasibility of carrying out this pilot study for systems vaccinology. Subjects were assigned to groups centrally through a balanced-block randomization process (random block size of 3,6 , or 9). The allocation schedule was computer generated in SAS 9.3 by an independent statistician who was not otherwise involved in planning or analysis. All subjects received their allocated vaccine on do (Figure 1) by the allocated route of administration to assess its immunogenicity and safety against A/California/7/2009 (H1N1)pdm09-like strain, A/Victoria/361/2011 (H3N2)-like strain, and B/Wisconsin/1/2010-like strain for the 2012-2013 season. The i.m. group received an injection of Vaxigrip (Sanofi Pasteur). The i.d. group received Intanza15 (Sanofi Pasteur) delivered via the BD Soluvia microinjector device. The t.c. group received Intanza15 by a t.c. needle-free method targeting hair follicles, according to the previously described standard operating procedure (10). All vaccines were administered in the deltoid region of the nondominant arm. Briefly, the t.c. vaccination area $(4 \times 4 \mathrm{~cm})$ was gently shaved, covered with a thin layer of cyanoacrylate (Superglue, UHU GmbH \& Co. KG), and then stripped with adhesive tape to open hair follicles. Vaccine was applied to the stripped skin inside a silicone barrier to limit the spread of the liquid, allowed to dry for 20 minutes, and then covered with a Comfeel adhesive bandage (Coloplast) for 24 hours. To measure humoral and cellular responses, blood and serum samples were collected from all subjects at visits on d0, d1, and d21.

The primary outcome was the $\mathrm{CD}^{+} \mathrm{T}$ cell response against the specific vaccine strain, measured at baseline and $\mathrm{d} 21$ after vaccination by 3 routes of immunization. Secondary outcomes were safety at each visit, measurement of microneutralizing antibodies and cellular responses at do, d21, and M5, and transcriptomic analysis at dO and d1. Immunomonitoring was performed blinded. Subjects used diary cards for 5 days after vaccination to report daily local reactions, systemic signs or symptoms, or ILIs. Data were collected in the electronic case report form. Neither any ILI nor any serious adverse event was reported during the study.

Hemagglutination inhibition and microneutralization assays. Serum antibodies against the 3 influenza viral strains contained in the 20122013 influenza vaccine were measured at d0, d21, and M5 with microtiter HI and MN assays. The method is described in Supplemental Materials and Methods.

Transcriptomic analysis. For transcriptomic analysis, $2.5 \mathrm{ml}$ whole blood was collected in PAXgene tubes (Qiagen) from each volunteer before and 1 day after vaccination. RNA was amplified with the Ovation Pico WTA system version 2 (NuGEN) and the resulting cDNA hybridized on Illumina whole human genome oligo microarrays (HT12 version 4.0). Genome Studio (Illumina) was used to analyze the data. Nonspecific background signal was filtered; quantile normalization of samples and $\log ^{2}$ transformation were applied to the gene raw data. In all, 47,323 probes were analyzed. 
Transcriptomic data were analyzed with $\mathrm{R}$ software 3.4 .1 (http://www.r-project.org/) and the limma package. Raw data were analyzed according to a linear model fit with a moderated $t$ test by empirical Bayes statistics. Adjusted $P$ values for simple multiple testing procedures were generated including Benjamini \& Yekutieli (2001) and Benjamini \& Hochberg (2006) procedures for the control of the false discovery rate (FDR). Corrected $P$ values less than 0.1 were considered to indicate statistical significance from transcriptomic data analyses for the 3 administration routes, and comparisons between $\mathrm{d} 0$ and $\mathrm{d} 1(\mathrm{~d} 1-\mathrm{d} 0)$. Statistical analyses and graphic representations were performed with Prism 6.0 (GraphPad Software Inc.). Comparisons between routes or between identified clusters used an unpaired Mann-Whitney $t$ test. All tests were 2sided. $P$ values less than 0.05 were used to identify genes with significant differential expression among vaccine responders (for the $\mathrm{d} 21 / \mathrm{d} 0$ ratio $\geq 4$ for $\mathrm{HI}$ and $\mathrm{MN}$ antibody titers and the $\mathrm{d} 21 / \mathrm{dO}$ ratio $\geq 2$ for $\mathrm{CD}^{+} \mathrm{GRZ}^{+} \mathrm{T}$ lymphocytes) and nonresponders. Correlations were identified with the Spearman coefficient, with significance defined by $P$ values less than 0.05 . Heatmaps and hierarchical clusters were generated with Multi Experiment Viewer MeV 4.9.0 (which is part of TM4 microarray software suite developed at The Institute for Genomic Research) (67). The Pearson coefficient of correlation with the complete linkage method generated hierarchical clusters, represented with dendograms, and R software was used to perform principal component analyses (PCA). Ingenuity pathway analysis (IPA) was used to perform functional enrichment analyses and identify new targets or candidate biomarkers within the context of biological systems. It provided the canonical pathways, molecular/ cellular functions, and networks that were statistically overrepresented in the gene signatures.

Statistics. Baseline characteristics of the study population were expressed as percentages for qualitative variables and as medians (with interquartile intervals) for continuous variables. Geometric means and 95\% CIs were calculated for $\mathrm{HI}$ and MN antibody titers. Intention-to-treat analyses were performed and included all randomized subjects. Characteristics were compared between the 3 groups with $\chi^{2}$ or Fisher's exact tests for qualitative variables and with Kruskal-Wallis or Mann-Whitney tests for quantitative variables. Immunological data analyses used samples from 60 subjects. Appropriate comparative statistics are stated in the text. Data were analyzed with SAS 9.3 and Graphpad Prism software version 6.0, with a 2-sided $P$ value of less than 0.05 considered statistically significant. Analysis of the difference between week 0 and week 14 within each individual group used a paired nonparameterized $t$ test (Wilcoxon matchedpairs signed rank test), with statistical significance set at $P$ less than 0.05. The logistic regression analyses and ROC curves were performed and generated with R.

Data availability statement. All data analyzed in this study are included in the published article. The normalized microarray data that support the finding of this study have been deposited in ArrayExpress with the accession code E-MTAB-7741.

Study approval. The FLUWAY original studies were conducted in accordance with the Declaration of Helsinki and the International Conference on Harmonization Good Clinical Practice guidelines and approved by relevant regulatory and independent ethics committees (Comité de Protection des Personnes, CPP Ile de France III Paris). Each participant provided written informed consent before study entry.

\section{Author contributions}

$\mathrm{EG}, \mathrm{OB}$, and AS contributed to data acquisition, analysis, interpretation, and drafted the work. PL was the clinical investigator and contributed to data collection. AR and MC contributed to the design, data management, data monitoring, and analysis. HB, RT, and NT contributed to transcriptomic data analysis. SB and SVW contributed to data collection. AV and TS were clinical investigators. OL was the principal clinical investigator of the FLUWAY study. BC designed the study, collected and analyzed data, and interpreted and wrote the manuscript.

\section{Acknowledgments}

We thank Sanofi-Pasteur (Marcy l'Etoile, France) for providing the Intanza 15 and Vaxigrip vaccines and note that they played no role in the design, management, or analysis of the study. We also thank the Sponsor, Assistance Publique - Hôpitaux de Paris (Département de la Recherche Clinique et du Développement, Clinical Research and Development Department). We are grateful to Melody Dufossé for technical assistance and to Lilia Ben-Slama and Hélène Perrin for project management. We thank Jo Ann Cahn for editing the English of our manuscript.

Address correspondence to: Béhazine Combadière, CimiParis/INSERM 1135, 91 Boulevard de l'Hôpital, 75013 Paris, France. Phone: 33140779888; Email: behazine.combadiere@ inserm.fr.
1. Combadiere B, Liard C. Transcutaneous and intradermal vaccination. Hum Vaccin. 2011;7(8):811-827.

2. Rappuoli R, Mandl CW, Black S, De Gregorio E. Vaccines for the twenty-first century society. Nat Rev Immunol. 2011;11(12):865-872.

3. Chen WH, et al. Safety, reactogenicity, and immunogenicity of inactivated monovalent influenza $\mathrm{A}(\mathrm{H} 5 \mathrm{~N} 1)$ virus vaccine administered with or without ASO3 adjuvant. Open Forum Infect Dis. 2014;1(3):ofu091.

4. Pileggi C, Lotito F, Bianco A, Nobile CG, Pavia M. Immunogenicity and safety of intradermal influenza vaccine in immunocompromized patients: a meta-analysis of randomized controlled trials. BMC Infect Dis. 2015;15:427.
5. Camilloni B, Basileo M, Valente S, Nunzi E, Iorio AM. Immunogenicity of intramuscular MF59adjuvanted and intradermal administered influenza enhanced vaccines in subjects aged over 60: a literature review. Hum Vaccin Immunother. 2015;11(3):553-563.

6. Liard C, et al. Targeting of HIV-p24 particlebased vaccine into differential skin layers induces distinct arms of the immune responses. Vaccine. 2011;29(37):6379-6391.

7. Liard C, et al. Intradermal immunization triggers epidermal Langerhans cell mobilization required for CD8 T-cell immune responses. J Invest Dermatol. 2012;132(3 Pt 1):615-625.

8. Belyakov IM, Hammond SA, Ahlers JD, Glenn GM, Berzofsky JA. Transcutaneous immuniza- tion induces mucosal CTLs and protective immunity by migration of primed skin dendritic cells. JClin Invest. 2004;113(7):998-1007.

9. Yagi $\mathrm{H}$, et al. Induction of therapeutically relevant cytotoxic T lymphocytes in humans by percutaneous peptide immunization. Cancer Res. 2006;66(20):10136-10144.

10. Vogt A, et al. Transcutaneous anti-influenza vaccination promotes both CD4 and CD8 T cell immune responses in humans. JImmunol. 2008;180(3):1482-1489.

11. Combadière B, et al. Preferential amplification of CD8 effector-T cells after transcutaneous application of an inactivated influenza vaccine: a randomized phase I trial. PLoS One. 2010;5(5):e10818. 
12. Fujiyama T, et al. Induction of cytotoxic T cells as a novel independent survival factor in malignant melanoma with percutaneous peptide immunization. J Dermatol Sci. 2014;75(1):43-48.

13. Vogt A, et al. $40 \mathrm{~nm}$, but not 750 or $1,500 \mathrm{~nm}$, nanoparticles enter epidermal Cd1a+ cells after transcutaneous application on human skin. J Invest Dermatol. 2006;126(6):1316-1322.

14. Henri S, et al. CD207+Cd103+ dermal dendritic cells cross-present keratinocyte-derived antigens irrespective of the presence of Langerhans cells. J Exp Med. 2010;207(1):189-206.

15. Levin C, et al. Critical role for skin-derived migratory DCs and Langerhans cells in TFH and GC responses after intradermal immunization. J Invest Dermatol. 2017;137(9):1905-1913.

16. Romani N, Flacher V, Tripp CH, Sparber F, Ebner $\mathrm{S}$, Stoitzner P. Targeting skin dendritic cells to improve intradermal vaccination. Curr Top Microbiol Immunol. 2012;351:113-138.

17. Hung IF, et al. Dose sparing intradermal trivalent influenza (2010/2011) vaccination overcomes reduced immunogenicity of the 2009 H1N1 strain. Vaccine. 2012;30(45):6427-6435.

18. Lambert PH, Laurent PE. Intradermal vaccine delivery: will new delivery systems transform vaccine administration? Vaccine. 2008;26(26):3197-3208.

19. Berkhoff EG, et al. Fitness costs limit escape from cytotoxic T lymphocytes by influenza A viruses. Vaccine. 2006;24(44-46):6594-6596.

20. La Gruta NL, Turner SJ. T cell mediated immunity to influenza: mechanisms of viral control. Trends Immunol. 2014;35(8):396-402.

21. Rimmelzwaan GF, Fouchier RA, Osterhaus AD. Influenza virus-specific cytotoxic T lymphocytes: a correlate of protection and a basis for vaccine development. Curr Opin Biotechnol. 2007;18(6):529-536.

22. Sridhar S, et al. Cellular immune correlates of protection against symptomatic pandemic influenza. Nat Med. 2013;19(10):1305-1312.

23. McMichael AJ, Gotch FM, Noble GR, Beare PA. Cytotoxic T-cell immunity to influenza. N Engl JMed.1983;309(1):13-17.

24. Bonduelle O, et al. Longitudinal and integrative biomodeling of effector and memory immune compartments after inactivated influenza vaccination. J Immunol. 2013;191(2):623-631.

25. Bonduelle O, et al. Characterization of pandemic influenza immune memory signature after vaccination or infection. J Clin Invest. 2014;124(7):3129-3136.

26. Pulendran B, Ahmed R. Translating innate immunity into immunological memory: implications for vaccine development. Cell. 2006;124(4):849-863.

27. Haining WN, Pulendran B. Identifying gnostic predictors of the vaccine response. Curr Opin Immunol. 2012;24(3):332-336

28. Bucasas KL, et al. Early patterns of gene expression correlate with the humoral immune response to influenza vaccination in humans. J Infect Dis. 2011;203(7):921-929.

29. Li S, et al. Molecular signatures of antibody responses derived from a systems biology study of five human vaccines. Nat Immunol.
2014;15(2):195-204.

30. Querec TD, et al. Systems biology approach predicts immunogenicity of the yellow fever vaccine in humans. Nat Immunol. 2009;10(1):116-125.

31. Rechtien A, et al. Systems vaccinology identifies an early innate immune signature as a correlate of antibody responses to the Ebola vaccine rVSV-ZEBOV. Cell Rep. 2017;20(9):2251-2261.

32. Haidari G, et al. Combined skin and muscle vaccination differentially impact the quality of effector T cell functions: the CUTHIVAC-001 randomized trial. Sci Rep. 2017;7(1):13011.

33. Nakaya HI, et al. Systems biology of vaccination for seasonal influenza in humans. Nat Immunol. 2011;12(8):786-795.

34. Quan FS, et al. Intradermal vaccination with influenza virus-like particles by using microneedles induces protection superior to that with intramuscular immunization.J Virol. 2010;84(15):7760-7769.

35. Sullivan SP, et al. Dissolving polymer microneedle patches for influenza vaccination. Nat Med. 2010;16(8):915-920.

36. Zhu W, et al. A boosting skin vaccination with dissolving microneedle patch encapsulating M2e vaccine broadens the protective efficacy of conventional influenza vaccines. J Control Release. 2017;261:1-9.

37. Boonnak K, et al. Immune responses to intradermal and intramuscular inactivated influenza vaccine among older age group. Vaccine. 2017;35(52):7339-7346

38. Camilloni B, Basileo M, Di Martino A, Donatelli I, Iorio AM. Antibody responses to intradermal or intramuscular MF59-adjuvanted influenza vaccines as evaluated in elderly institutionalized volunteers during a season of partial mismatching between vaccine and circulating $\mathrm{A}(\mathrm{H} 3 \mathrm{~N} 2)$ strains. Immun Ageing. 2014;11:10.

39. Holland D, et al. Intradermal influenza vaccine administered using a new microinjection system produces superior immunogenicity in elderly adults: a randomized controlled trial. JInfect Dis. 2008;198(5):650-658.

40. Tsang P, et al. Immunogenicity and safety of Fluzone $\left({ }^{\circledR}\right)$ intradermal and high-dose influenza vaccines in older adults $\geq 65$ years of age: a randomized, controlled, phase II trial. Vaccine. 2014;32(21):2507-2517.

41. Gorse GJ, et al. Safety and immunogenicity of revaccination with reduced dose intradermal and standard dose intramuscular influenza vaccines in adults 18-64 years of age. Vaccine. 2013;31(50):6034-6040.

42. Nakaya HI, Pulendran B. Systems vaccinology: its promise and challenge for HIV vaccine development. Curr Opin HIV AIDS. 2012;7(1):24-31.

43. Pulendran B. Learning immunology from the yellow fever vaccine: innate immunity to systems vaccinology. Nat Rev Immunol. 2009;9(10):741-747.

44. Nakaya HI, Li S, Pulendran B. Systems vaccinology: learning to compute the behavior of vaccine induced immunity. Wiley Interdiscip Rev Syst Biol Med. 2012;4(2):193-205.

45. Pulendran B, Li S, Nakaya HI. Systems vaccinology.
Immunity. 2010;33(4):516-529.

46. Gaucher D, et al. Yellow fever vaccine induces integrated multilineage and polyfunctional immune responses. J Exp Med. 2008;205(13):3119-3131.

47. Kees U, Krammer PH. Most influenza A virus-specific memory cytotoxic T lymphocytes react with antigenic epitopes associated with internal virus determinants. J Exp Med. 1984;159(2):365-377.

48. Zweerink HJ, Courtneidge SA, Skehel JJ, Crumpton MJ, Askonas BA. Cytotoxic T cells kill influenza virus infected cells but do not distinguish between serologically distinct type A viruses. Nature. 1977;267(5609):354-356

49. Shi L, et al. Over-expression of CKS1B activates both MEK/ERK and JAK/STAT3 signaling pathways and promotes myeloma cell drugresistance. Oncotarget. 2010;1(1):22-33.

50. Carroll MC. Complement and humoral immunity. Vaccine. 2008;26 Suppl 8:I28-I33.

51. Martin C, Burdon PC, Bridger G, GutierrezRamos JC, Williams TJ, Rankin SM. Chemokines acting via CXCR2 and CXCR4 control the release of neutrophils from the bone marrow and their return following senescence. Immunity. 2003;19(4):583-593.

52. Yuan J, Zhang Y, Yan FT, Zheng X. Association of PRKAA1 gene polymorphisms with chronic hepatitis B virus infection in Chinese Han population. Braz J Infect Dis. 2016;20(6):564-568.

53. Prantner D, Perkins DJ, Vogel SN. AMP-activated kinase (AMPK) promotes innate immunity and antiviral defense through modulation of stimulator of interferon genes (STING) signaling. J Biol Chem. 2017;292(1):292-304.

54. He S, et al. Tumor suppressor NGX6 inhibits the growth and metastasis of multiple cancers. Tumour Biol. 2016;37(5):5751-5760.

55. Chiang CY, et al. Cofactors required for TLR7and TLR9-dependent innate immune responses. Cell Host Microbe. 2012;11(3):306-318.

56. Nie Y, Waite J, Brewer F, Sunshine MJ, Littman DR, Zou YR. The role of CXCR4 in maintaining peripheral B cell compartments and humoral immunity. J Exp Med. 2004;200(9):1145-1156.

57. Eash KJ, Means JM, White DW, Link DC. CXCR4 is a key regulator of neutrophil release from the bone marrow under basal and stress granulopoiesis conditions. Blood. 2009;113(19):4711-4719.

58. Carty M, Goodbody R, Schröder M, Stack J, Moynagh PN, Bowie AG. The human adaptor SARM negatively regulates adaptor protein TRIF-dependent Toll-like receptor signaling. Nat Immunol. 2006;7(10):1074-1081.

59. Furman D, et al. Apoptosis and other immune biomarkers predict influenza vaccine responsiveness. Mol Syst Biol. 2013;9:659.

60. Franco LM, et al. Integrative genomic analysis of the human immune response to influenza vaccination. Elife. 2013;2:e00299.

61. Daudelin JF, Mathieu M, Boulet S, Labrecque N. IL-6 production by dendritic cells is dispensable for CD8+ memory T-cell generation. Biomed Res Int. 2013;2013:126189.

62. Duffy D, et al. Neutrophils transport antigen 
from the dermis to the bone marrow, initiating a source of memory CD8+ T cells. Immunity. 2012;37(5):917-929.

63. Gonnet J, et al. Interleukin-32 promotes detachment and activation of human Langerhans cells in a human skin explant model. Br J Dermatol. 2018;179(1):145-153.
64. MacLeod MK, et al. Vaccine adjuvants aluminum and monophosphoryl lipid A provide distinct signals to generate protective cytotoxic memory CD8 T cells. Proc Natl Acad Sci U S A. 2011;108(19):7914-7919.

65. Tsang JS. Utilizing population variation, vaccination, and systems biology to study human immu- nology. Trends Immunol. 2015;36(8):479-493. 66. Tsang JS, et al. Global analyses of human immune variation reveal baseline predictors of postvaccination responses. Cell. 2014;157(2):499-513.

67. Saeed AI, et al. TM4: a free, open-source system for microarray data management and analysis. BioTechniques. 2003;34(2):374-378. 